\title{
The genetic background of the associations between sense of coherence and mental health, self-esteem and personality
}

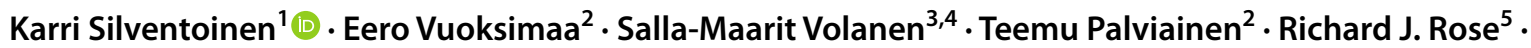 \\ Sakari Suominen $^{6,7} \cdot$ Jaakko Kaprio $^{2}$
}

Received: 19 January 2021 / Accepted: 23 April 2021 / Published online: 19 May 2021

(c) The Author(s) 2021

\begin{abstract}
Purpose Sense of coherence (SOC) represents coping and can be considered an essential component of mental health. SOC correlates with mental health and personality, but the background of these associations is poorly understood. We analyzed the role of genetic factors behind the associations of SOC with mental health, self-esteem and personality using genetic twin modeling and polygenic scores (PGS).

Methods Information on SOC (13-item Orientation of Life Questionnaire), four mental health indicators, self-esteem and personality (NEO Five Factor Inventory Questionnaire) was collected from 1295 Finnish twins at 20-27 years of age.

Results In men and women, SOC correlated negatively with depression, alexithymia, schizotypal personality and overall mental health problems and positively with self-esteem. For personality factors, neuroticism was associated with weaker SOC and extraversion, agreeableness and conscientiousness with stronger SOC. All these psychological traits were influenced by genetic factors with heritability estimates ranging from 19 to $66 \%$. Genetic and environmental factors explained these associations, but the genetic correlations were generally stronger. The PGS of major depressive disorder was associated with weaker, and the PGS of general risk tolerance with stronger SOC in men, whereas in women the PGS of subjective well-being was associated with stronger SOC and the PGSs of depression and neuroticism with weaker SOC.

Conclusion Our results indicate that a substantial proportion of genetic variation in SOC is shared with mental health, selfesteem and personality indicators. This suggests that the correlations between these traits reflect a common neurobiological background rather than merely the influence of external stressors.
\end{abstract}

Keywords Sense of coherence $\cdot$ Mental health $\cdot$ Personality $\cdot$ Twins $\cdot$ Polygenic score

Karri Silventoinen

karri.silventoinen@helsinki.fi

1 Faculty of Social Sciences, Department of Social Research, University of Helsinki, P.O. Box 18, N00014 Helsinki, Finland

2 Institute for Molecular Medicine Finland (FIMM), HiLIFE, University of Helsinki, Helsinki, Finland

3 Folkhälsan Research Center, Helsinki, Finland

4 Clinicum, Department of Public Health, University of Helsinki, Helsinki, Finland

5 Department of Psychological \& Brain Sciences, Indiana University, Bloomington, IN, USA

6 Department of Public Health, University of Turku, Turku, Finland

7 School of Health Sciences, University of Skövde, Skövde, Sweden

\section{Introduction}

While mental health and well-being are related and even used interchangeably, mental health is generally taken to refer to the presence of psychiatric illness or psychopathology, whereas well-being is about positive psychological health. Thus, a state of health may be regarded not only as the absence of disease but can also cover positive qualities such as life satisfaction, motivation and a sense of fulfilment. Well-being is often characterized by two components: (i) "feeling well" (i.e., hedonic well-being, covering overall interest in life, happiness and life satisfaction) and (ii) "doing well" (i.e., eudaemonic well-being, which represents a more cognitive appraisal of one's relationships, personal growth and optimal functioning) [1]. The salutogenic approach to well-being relates to "doing well" by emphasizing resources helping to cope with external stressors and 
factors supporting mental health [2]. A key concept in the salutogenic theory is the sense of coherence (SOC): a life orientation enabling coping in arduous life situations and thus staying healthy regardless of the presence of external stressors. A strong SOC allows for seeing the world as comprehensible, meaningful and manageable, reflecting the three main dimensions of SOC. Previous studies have shown that SOC is associated with better mental and physical health [3] and a better quality of life as predicted by the theory [4]. Further, high internal validity of SOC has been shown in previous studies, as well as moderate to high test-retest correlations, at least within the time frame of a few years, showing the consistency of SOC [5].

Although it has been proposed that SOC represents a fundamentally different approach to mental health measuring the capacity to maintain health (salutogenesis) in contrast to the classical focus on risks and diseases (pathogenesis) [6], previous research has challenged this distinction. There is evidence that SOC is inversely associated with indicators of mental health problems, such as depression and anxiety $[7,8]$. It is possible that SOC measures the same dimension of mental health measured by instruments focused on identifying psychiatric diseases and mental health problems. Further, associations have been found between SOC and personality factors: according to a recent meta-analysis of 19 studies using the NEO Five Factor Inventory (FFI) personality scale, neuroticism was associated with weaker and extraversion, openness, agreeableness and conscientiousness with stronger SOC [9].

A limitation in the previous studies on the associations of SOC with mental health and personality indicators is that they have not considered whether the same environmental factors can generally be behind these associations. For example, if the same external exposure, known to be negatively associated with SOC, such as negative life events [10] or work-related stress [11], also affects mental health, it can create a correlation between these traits. These effects can be reinforced since many studies of SOC have been conducted in patients or other populations with special external stressors [3, 4]. Similarly, shared genetic effects may underlie the associations of SOC with other traits. It has been shown that many psychiatric disorders show substantial genetic correlations based on measured genetic variants $[12,13]$, suggesting that a shared genetic liability may exist. Measures of mental well-being also show substantial heritability in twin and family studies, and some large molecular genetics studies of well-being have been conducted $[14,15]$. Nonetheless, progress in identifying the actual genetic basis of well-being is much slower than for specific mental disorders. Thus, it is possible that some of the same genes that explain individual differences in SOC are related to individual differences in mental health problems. Molecular genetic studies of SOC are, however, still lacking.
In this study, we aim to obtain more information on these associations by studying the genetic correlations between SOC, mental health, self-esteem and personality. We will use two approaches to estimate the common genetic background, each making different theoretical assumptions: (i) a classical twin design utilizing the genetic similarity of twins and (ii) a molecular genetic design utilizing measured information on genetic polymorphisms through the whole genome.

\section{Data and methods}

We used data derived from the FinnTwin12 study having the target population of all Finnish twins born in 1983-1987 $(N=6272)$ [16]. From the Finnish population registry (which covers the entire population), the twins were identified as those born on the same day to the same mother. The baseline postal questionnaire was sent to the twins when they were 11 to 12 years of age, and 4920 twins responded (78\% of all twins in the cohort). This current study is based on the fourth wave of the FinnTwin12 study, when the twins were 20-27 years of age. During this time, a sub-cohort of twins were invited to an intensive study when information on SOC, mental health, self-esteem and personality was collected by a questionnaire, and they also gave a DNA sample [17]. Together, 1852 twins were invited to this intensive study and 1295 twins returned the questionnaire (54\% females). After removing two twin individuals with unknown zygosity, we had 254 complete monozygotic (MZ), 176 same-sex dizygotic (SSDZ), and 156 opposite-sex dizygotic (OSDZ) pairs informing the genetic twin modeling. Twins without information on their co-twin $(N=121)$ were removed from the genetic twin analyses. Further, we had genotypic data available for 1257 twin individuals used in the molecular genetic analyses. For twins with a DNA sample, zygosity was based on measured genotypes (i.e., single-nucleotide polymorphisms (SNP)), and for the few twins who did not give a DNA sample, zygosity was based on questions of physical similarity in the baseline questionnaire, a method that has shown high reliability in this cohort [18]. The number of missing observations for individual items was small $(N=41$ for schizotypal personality and a maximum of five for other traits).

SOC was measured with the Antonovsky's 13-item short scale derived from the original 29-item Orientation to Life Questionnaire [2]. The mean score of all items was used as a measure of SOC. The Cronbach's $\alpha$ for SOC in our data was 0.85 , showing good internal consistency of the scale. We decided to only report the results for general SOC because it correlated strongly with all three dimensions of SOC ( $r=0.89$ for comprehensibility, $r=0.86$ for manageability and $r=0.80$ for meaningfulness after adjusting for 
sex) in this sample. However, to confirm that the different dimensions of SOC were not differently associated with other psychological traits, we calculated the trait correlations of them with mental health indicators, self-esteem and personality traits. The heritability estimates for SOC and its three components have previously been reported in a larger cohort that included also twin pairs used in this study [19].

Mental health was measured with four scales described in detail elsewhere. Cronbach's $\alpha$ values calculated for mental health and self-esteem varied from 0.80 to 0.91 , showing good to excellent internal consistency of the scales. Depressive symptoms were measured by the 10 -item short version of the General Behavior Inventory questionnaire on mood-related behaviors such as depressive, hypomanic and biphasic symptoms, which was also used in previous Finnish studies $(\alpha=0.91)$ [20]. Alexithymia was measured by the 20-item Toronto Alexithymia Scale measuring problems of describing and identifying emotions [21]. A sum score with a possible range of $20-100$ was used $(\alpha=0.83)$. Schizotypal personality was measured by the 22 -item Schizotypal Personality Questionnaire-Brief $(\alpha=0.80)$ [22]. Overall mental health problems were measured with the Goldberg 12-item General Health Questionnaire (GHQ) asking about issues affecting general mood and mental health problems in everyday life ( $\alpha=0.87$ ) [23]. Self-esteem was assessed by the 10-item Rosenberg Self-Esteem Scale, a uni-dimensional measure of global self-esteem measuring overall feelings of self-worth and self-acceptance [24] and previously used in studies based on Finnish twin data [25]. We used the sum score with a possible range of $10-40(\alpha=0.89)$. Personality was measured with the 55-item NEO FFI that yielded mean scores in five personality traits: neuroticism, extraversion, openness, agreeableness and conscientiousness [26]. The Finnish version of the NEO FFI is based on a longer 180-item personality inventory, which is an authorized adaptation of the NEO Personality Inventory [27]. Seven extra items for a sensation-seeking facet of the extraversion scale were also used in our study.

We started the analyses by examining genetic and environmental factors affecting SOC, mental health, self-esteem and personality using a quantitative genetic twin design based on the comparisons of similarity between MZ and DZ twins [28]. MZ twins are virtually genetically identical at the gene sequence level whereas DZ twins share half of their genetic variation, similar to ordinary siblings. Both MZ and DZ twins are assumed to share the same amount of environmental variation. Based on these principles, the trait variation can be decomposed to additive genetic variation (A) including all main effects of the loci affecting the trait (correlations of 1.0 within $\mathrm{MZ}$ and 0.5 within $\mathrm{DZ}$ pairs); dominance genetic variation (D) caused by interactions between alleles in the same locus (correlations of 1.0 within MZ and 0.25 within DZ pairs); shared environment
(C) including the effect of all environmental factors that make twins in a pair similar to each other (correlations of 1.0 within both $\mathrm{MZ}$ and $\mathrm{DZ}$ twins); and unique environment (E) including environmental factors specific to each twin individual as well as any measurement error (correlation of 0 within both $\mathrm{MZ}$ and $\mathrm{DZ}$ twins). Because we have only twins reared together in our data, we were unable to estimate $\mathrm{D}$ and $\mathrm{C}$ effects simultaneously.

Univariate models were used to test the assumptions of twin modeling (i.e., the same means and standard deviations (SD) for first- and second-born twins as well as MZ and DZ twins), finding the best fitting model, as well as to estimate the heritability components (i.e., the proportion of total variation explained by genetic variation) under the best fitting model. Since age was not correlated with SOC, the mental health indicators, self-esteem or the personality factors $(r=-0.06-0.05 ; p$ values $\geq 0.064)$, we did not adjust the results for age. Model fit statistics are presented in Supplementary Table 1 . We first estimated the additive genetic/ shared environment/unique environment (ACE) model and the additive genetic/dominance genetic/unique environment (ADE) model. When we compared a more parsimonious additive genetic/unique environment (AE) model to the better fitting model (ADE or ACE model having a lower - 2 log likelihood value), we found that the decrease of model fit was not statistically significant ( $p \geq 0.344$ ), suggesting that shared environment and dominance genetic factors were not needed in the model and could be constrained to zero. Thus, the model used in our study makes assumptions that all effects of alleles on the psychological traits are additive and there are no environmental factors shared by co-twins affecting these traits. Sex-specific genetic factors were statistically significant for four traits and the size of variance components showed statistically significant differences between men and women for six traits. When the full $\mathrm{AE}$ model was compared with the saturated model, a violation of the assumptions of twin modeling was found for depression $(p=0.050)$, overall mental health problems $(p<0.001)$, and self-esteem $(p=0.035)$. However, if using the Bonferronicorrected $\mathrm{p}$ values for multiple testing ( $p=0.0045$ for 11 tests based on the conventional significance level of 0.05 ), the violation was statistically significant only for overall mental health problems. Thus, we used the full AE model to (i) estimate the proportion of variation of SOC, mental health indicators, self-esteem and personality components explained by additive genetic and unique environmental factors using a univariate model and then (ii) calculated how much these factors explained the associations of SOC with the other psychological measures using Cholesky decomposition. This method decomposes all variation and co-variation into uncorrelated latent factors.

We continued the study by analyzing how SOC correlated with the polygenic risk scores (PGS) of the following 
six traits: major depressive disorder, general risk tolerance, schizophrenia, depression, neuroticism and subjective well-being. The technical details of genotyping have been described elsewhere [29]. To obtain PGSs, we implemented a Bayesian approach taking into account the linkage disequilibrium between each variant [30]. The model for calculating PGSs was adjusted for a linkage disequilibrium reference panel consisting of 27,284 unrelated Finnish samples from the national FINRISK study [31]. Genome-wide-association (GWA) summary statistics for the PGS calculations were obtained from the Psychiatric Genomics Consortium website (https://www.med.unc.edu/pgc/) for major depression [32] and schizophrenia [33], from the Social Science Genetic Association Consortium website (https://www.thess gac.org/data) for general risk tolerance [34] and subjective well-being [14] and from the Neale lab repository of UK Biobank summary statistics for depression and neuroticism scores (http://www.nealelab.is/uk-biobank). The total number of SNPs used for PGS calculations were 1,147,810 for major depression, 1,144,587 for schizophrenia, 1,147,378 for general risk tolerance, 99,7410 for subjective well-being, $1,142,239$ for broad depression and 1,142,239 for neuroticism scores. We calculated standardized $\beta$-coefficients presenting the change of SOC score per the change of 1 SD of each PGS as well as the proportion of SOC variance explained by each PGS $\left(R^{2}\right)$.

Finally, we conducted a GWA analysis for SOC using the following procedure: genotypes were imputed to the TOPMed release 2 reference panel [35] using minimac4 [36] in the TOPMed imputation server [37] and then we performed association analysis using linear mixed models using age, sex and eight genetic principal components as covariates with an empirical kinship matrix in the random effect of the model controlling for familial and more distant genetic relatedness. The association testing was performed using score tests using genotype dosages for alternate alleles. The analysis was performed using the RVTESTS package [37]. After the analysis, we filtered out all variants with alternate an allele frequency $<1 \%$, HWE $p$ value $<1 \mathrm{e}-06$ and imputation quality $<0.8$.

The genetic twin models were fitted using the OpenMx package, version 3.0.2, of $\mathrm{R}$ statistical software [38]. The PGS analyses as well as the statistical tests for all descriptive statistics were performed using linear regression models by the Stata/SE 16.1 for Windows statistical software (StataCorp, College Station, TX, USA) using the cluster option to correct the standard errors and confidence intervals (CI) for the lack of statistical independence of twins sampled as twin pairs [39].

\section{Results}

Table 1 presents the descriptive statistics for SOC, mental health indicators, self-esteem and personality traits by sex. Men had stronger SOC and generally better mental health and self-esteem than women. The only exception was alexithymia, with men reporting more problems in identifying emotions. In the personality traits, men showed a higher level of extraversion whereas women had higher levels of neuroticism, openness and agreeableness. In conscientiousness, no sex difference was found.

Table 2 presents the proportions of total variance decomposed to additive genetic and unique environmental variances for all psychological traits. The results showed moderate heritability estimates varying between 0.19 and 0.66 . For most of the traits, men showed lower heritabilities than women. Even when the $95 \%$ CIs were overlapping, the
Table 1 Descriptive statistics of sense of coherence, mental health, self-esteem and personality factors by sex

\begin{tabular}{|c|c|c|c|c|c|c|c|}
\hline & \multicolumn{3}{|l|}{ Men } & \multicolumn{3}{|c|}{ Women } & \multirow{2}{*}{$\begin{array}{l}p \text {-value of } \\
\text { sex differ- } \\
\text { ence }\end{array}$} \\
\hline & $N$ & Mean & SD & $N$ & Mean & SD & \\
\hline Sense of coherence & 594 & 64.1 & 10.00 & 700 & 61.7 & 10.63 & $<0.0001$ \\
\hline \multicolumn{8}{|l|}{ Mental health } \\
\hline Depression & 593 & 13.6 & 4.10 & 699 & 15.3 & 4.94 & $<0.0001$ \\
\hline Alexithymia & 594 & 29.7 & 9.42 & 700 & 27.8 & 10.29 & 0.002 \\
\hline Schizotypal personality & 576 & 5.2 & 4.32 & 677 & 6.1 & 4.42 & 0.001 \\
\hline Overall mental health problems & 591 & 21.3 & 4.24 & 699 & 23.5 & 5.52 & $<0.0001$ \\
\hline Self-esteem & 593 & 33.2 & 4.94 & 699 & 30.4 & 5.54 & $<0.0001$ \\
\hline \multicolumn{8}{|l|}{ Personality } \\
\hline Neuroticism & 595 & 1.4 & 0.63 & 700 & 1.9 & 0.70 & $<0.0001$ \\
\hline Extraversion & 595 & 2.5 & 0.46 & 700 & 2.3 & 0.41 & $<0.0001$ \\
\hline Openness & 595 & 2.0 & 0.54 & 700 & 2.2 & 0.51 & $<0.0001$ \\
\hline Agreeableness & 595 & 2.6 & 0.42 & 700 & 2.7 & 0.47 & 0.010 \\
\hline Conscientiousness & 595 & 2.5 & 0.53 & 700 & 2.6 & 0.54 & 0.218 \\
\hline
\end{tabular}


Table 2 The proportion of variation of sense of coherence, mental health, self-esteem and personality indicators explained by additive genetic and unique environmental factors by sex

\begin{tabular}{|c|c|c|c|c|c|c|}
\hline & \multicolumn{3}{|c|}{ Additive genetic factors } & \multicolumn{3}{|c|}{ Unique environmental factors } \\
\hline & \multirow[t]{2}{*}{$a^{2}$} & \multicolumn{2}{|c|}{$95 \% \mathrm{CI}$} & \multirow[t]{2}{*}{$\mathrm{e}^{2}$} & \multicolumn{2}{|c|}{$95 \% \mathrm{CI}$} \\
\hline & & LL & UL & & LL & UL \\
\hline \multicolumn{7}{|l|}{ Men } \\
\hline Sense of coherence & 0.30 & 0.15 & 0.44 & 0.70 & 0.56 & 0.85 \\
\hline \multicolumn{7}{|l|}{ Mental health } \\
\hline Depression & 0.37 & 0.21 & 0.51 & 0.63 & 0.49 & 0.79 \\
\hline Alexithymia & 0.44 & 0.29 & 0.56 & 0.56 & 0.44 & 0.71 \\
\hline Schizotypal personality & 0.52 & 0.38 & 0.63 & 0.48 & 0.37 & 0.62 \\
\hline Overall mental health problems & 0.19 & 0.02 & 0.35 & 0.81 & 0.65 & 0.98 \\
\hline Self-esteem & 0.35 & 0.18 & 0.49 & 0.65 & 0.51 & 0.82 \\
\hline \multicolumn{7}{|l|}{ Personality } \\
\hline Neuroticism & 0.43 & 0.29 & 0.55 & 0.57 & 0.45 & 0.71 \\
\hline Extraversion & 0.60 & 0.48 & 0.69 & 0.40 & 0.31 & 0.52 \\
\hline Openness & 0.66 & 0.55 & 0.74 & 0.34 & 0.26 & 0.45 \\
\hline Agreeableness & 0.24 & 0.09 & 0.38 & 0.76 & 0.62 & 0.91 \\
\hline Conscientiousness & 0.51 & 0.37 & 0.62 & 0.49 & 0.38 & 0.63 \\
\hline \multicolumn{7}{|l|}{ Women } \\
\hline Sense of coherence & 0.51 & 0.39 & 0.61 & 0.49 & 0.39 & 0.61 \\
\hline \multicolumn{7}{|l|}{ Mental health } \\
\hline Depression & 0.63 & 0.52 & 0.71 & 0.37 & 0.29 & 0.48 \\
\hline Alexithymia & 0.41 & 0.29 & 0.52 & 0.59 & 0.48 & 0.71 \\
\hline Schizotypal personality & 0.57 & 0.47 & 0.66 & 0.43 & 0.34 & 0.53 \\
\hline Overall mental health problems & 0.40 & 0.26 & 0.51 & 0.60 & 0.49 & 0.74 \\
\hline Self-esteem & 0.59 & 0.49 & 0.68 & 0.41 & 0.32 & 0.51 \\
\hline \multicolumn{7}{|l|}{ Personality } \\
\hline Neuroticism & 0.58 & 0.47 & 0.66 & 0.42 & 0.34 & 0.53 \\
\hline Extraversion & 0.56 & 0.46 & 0.65 & 0.44 & 0.35 & 0.54 \\
\hline Openness & 0.61 & 0.51 & 0.69 & 0.39 & 0.31 & 0.49 \\
\hline Agreeableness & 0.57 & 0.46 & 0.66 & 0.43 & 0.34 & 0.54 \\
\hline Conscientiousness & 0.54 & 0.42 & 0.64 & 0.46 & 0.36 & 0.58 \\
\hline
\end{tabular}

model fit statistics showed that the magnitude of genetic effects differed between men and women in depression, overall mental health problems, self-esteem, neuroticism, extraversion and agreeableness (Supplementary Table 1).

The trait correlations of SOC with the other psychological traits as well as the decomposition of these trait correlations to additive genetic and unique environmental correlations are presented in Table 3. Those with stronger SOC had lower levels of depression, alexithymia, schizotypal personality and overall mental health problems and better self-esteem. Generally, the correlations were very similar in men and women. When these trait correlations were decomposed, both additive genetic and unique environmental factors explained a part of them. However, additive genetic correlations were, with a few exceptions, substantially stronger than unique environmental correlations. The strongest genetic correlations were found for depression $\left(r_{\mathrm{A}}=-0.82\right.$ in men and -0.84 women), showing that $67 \%$ of genetic variation in men and $71 \%$ in women was shared with SOC; in females the genetic correlation for overall mental health problems was also high $\left(r_{\mathrm{A}}=-0.84\right)$. However, all genetic correlations were at least moderate, showing that the traits share $17 \%$ or more common genetic variation with SOC.

When studying the personality traits, those men and women with stronger SOC showed a lower level of neuroticism but higher levels of extraversion, agreeableness and conscientiousness. For openness, no association with SOC was found. As for the mental health traits and self-esteem, genetic correlations were higher than unique environmental correlations even when both of these components explained a share of co-variation between SOC and personality. The highest genetic correlation of SOC was found with neuroti$\operatorname{cism}\left(r_{\mathrm{A}}=-0.78\right.$ in men and $r_{\mathrm{A}}=-0.85$ in women $)$ showing that $61 \%$ of genetic variation in men and $72 \%$ in women was shared between these traits. When we tested the associations of mental health traits, self-esteem and personality factors 
Table 3 Trait correlations of sense of coherence with mental health, self-esteem and personality factors as well as additive genetic and unique environmental correlations explaining these trait correlations by sex

\begin{tabular}{|c|c|c|c|c|c|c|c|c|c|c|c|}
\hline & \multicolumn{3}{|c|}{ Trait correlation } & \multicolumn{4}{|c|}{ Additive genetic correlation } & \multicolumn{4}{|c|}{ Unique environmental correlation } \\
\hline & \multirow[t]{2}{*}{$r$} & \multicolumn{2}{|c|}{$95 \% \mathrm{CI}$} & \multirow[t]{2}{*}{$r_{\mathrm{A}}$} & \multicolumn{2}{|l|}{$95 \% \mathrm{CI}$} & \multirow[t]{2}{*}{$\%$ explained } & \multirow[t]{2}{*}{$r_{\mathrm{E}}$} & \multicolumn{2}{|l|}{$95 \% \mathrm{CI}$} & \multirow[t]{2}{*}{$\%$ explained } \\
\hline & & LL & UL & & LL & UL & & & LL & UL & \\
\hline \multicolumn{12}{|l|}{ Men } \\
\hline \multicolumn{12}{|l|}{ Mental health } \\
\hline Depression & -0.58 & -0.63 & -0.52 & -0.82 & -1.00 & -0.60 & 0.48 & -0.46 & -0.57 & -0.32 & 0.52 \\
\hline Alexithymia & -0.50 & -0.57 & -0.43 & -0.71 & -0.98 & -0.46 & 0.51 & -0.39 & -0.52 & -0.25 & 0.49 \\
\hline Schizotypal personality & -0.61 & -0.66 & -0.55 & -0.65 & -0.83 & -0.45 & 0.43 & -0.61 & -0.70 & -0.49 & 0.57 \\
\hline Overall mental health problems & -0.47 & -0.53 & -0.40 & -0.41 & -0.85 & 0.34 & 0.18 & -0.49 & -0.59 & -0.36 & 0.82 \\
\hline Self-esteem & 0.62 & 0.56 & 0.67 & 0.53 & 0.13 & 0.73 & 0.28 & 0.66 & 0.56 & 0.74 & 0.72 \\
\hline \multicolumn{12}{|l|}{ Personality } \\
\hline Neuroticism & -0.69 & -0.73 & -0.64 & -0.78 & -0.93 & -0.59 & 0.41 & -0.65 & -0.73 & -0.54 & 0.59 \\
\hline Extraversion & 0.32 & 0.24 & 0.39 & 0.39 & 0.13 & 0.63 & 0.52 & 0.29 & 0.13 & 0.44 & 0.48 \\
\hline Openness & -0.07 & -0.16 & 0.02 & -0.15 & -0.41 & 0.11 & 0.99 & 0.00 & -0.17 & 0.17 & 0.01 \\
\hline Agreeableness & 0.41 & 0.33 & 0.48 & 0.59 & 0.19 & 0.96 & 0.34 & 0.36 & 0.22 & 0.48 & 0.66 \\
\hline Conscientiousness & 0.35 & 0.27 & 0.43 & 0.60 & 0.34 & 0.87 & 0.66 & 0.21 & 0.05 & 0.36 & 0.34 \\
\hline \multicolumn{12}{|l|}{ Women } \\
\hline \multicolumn{12}{|l|}{ Mental health } \\
\hline Depression & -0.66 & -0.71 & -0.61 & -0.84 & -0.93 & -0.75 & 0.72 & -0.43 & -0.55 & -0.30 & 0.28 \\
\hline Alexithymia & -0.53 & -0.59 & -0.47 & -0.66 & -0.79 & -0.50 & 0.56 & -0.43 & -0.54 & -0.30 & 0.43 \\
\hline Schizotypal personality & -0.62 & -0.67 & -0.57 & -0.79 & -0.89 & -0.68 & 0.69 & -0.42 & -0.54 & -0.30 & 0.31 \\
\hline Overall mental health problems & -0.55 & -0.60 & -0.49 & -0.84 & -0.98 & -0.69 & 0.69 & -0.31 & -0.44 & -0.18 & 0.31 \\
\hline Self-esteem & 0.62 & 0.57 & 0.67 & 0.72 & 0.61 & 0.83 & 0.66 & 0.51 & 0.39 & 0.61 & 0.34 \\
\hline \multicolumn{12}{|l|}{ Personality } \\
\hline Neuroticism & -0.72 & -0.76 & -0.68 & -0.85 & -0.93 & -0.76 & 0.63 & -0.59 & -0.68 & -0.48 & 0.37 \\
\hline Extraversion & 0.17 & 0.09 & 0.25 & 0.12 & -0.07 & 0.30 & 0.37 & 0.23 & 0.08 & 0.37 & 0.63 \\
\hline Openness & -0.07 & -0.15 & 0.01 & -0.17 & -0.35 & 0.01 & 1.31 & 0.05 & -0.10 & 0.20 & -0.31 \\
\hline Agreeableness & 0.42 & 0.35 & 0.49 & 0.70 & 0.55 & 0.85 & 0.88 & 0.11 & -0.04 & 0.25 & 0.12 \\
\hline Conscientiousness & 0.33 & 0.25 & 0.40 & 0.52 & 0.34 & 0.70 & 0.81 & 0.13 & -0.03 & 0.28 & 0.19 \\
\hline
\end{tabular}

separately with the three dimensions of SOC, we found that the correlations were very similar for comprehensibility, manageability and meaningfulness (Supplementary Table 2).

Table 4 presents the associations of the PGSs of three mental health disorders, general risk tolerance, subjective well-being and neuroticism with SOC. In men, the PGS of major depressive disorders was associated with weaker and general risk tolerance with stronger SOC; for schizophrenia a negative association was found, but it was only marginally significant. In women, the PGSs of depression and neuroticism were associated with weaker and subjective well-being with stronger SOC. We also tested the sex interactions for all
Table 4 The standardized regression coefficients and the proportions of variation explained by polygenic scores for sense of coherence

\begin{tabular}{|c|c|c|c|c|c|c|c|c|}
\hline & \multicolumn{4}{|l|}{ Men } & \multicolumn{4}{|c|}{ Women } \\
\hline & \multirow[t]{2}{*}{$\beta$} & \multicolumn{2}{|l|}{$95 \% \mathrm{CI}$} & \multirow[t]{2}{*}{$R^{2}$} & \multirow[t]{2}{*}{$\beta$} & \multicolumn{2}{|l|}{$95 \% \mathrm{CI}$} & \multirow[t]{2}{*}{$R^{2}$} \\
\hline & & LL & UL & & & LL & UL & \\
\hline Major depressive disorder & -1.06 & -1.97 & -0.16 & 0.06 & -0.72 & -1.53 & 0.09 & 0.03 \\
\hline General risk tolerance & 1.03 & 0.19 & 1.86 & 0.06 & -0.39 & -1.24 & 0.45 & 0.03 \\
\hline Schizophrenia & -0.81 & -1.62 & 0.01 & 0.05 & -0.09 & -1.00 & 0.81 & 0.02 \\
\hline Depression & 0.17 & -0.68 & 1.01 & 0.05 & -0.82 & -1.59 & -0.06 & 0.03 \\
\hline Neuroticism & -0.26 & -1.14 & 0.61 & 0.05 & -0.86 & -1.67 & -0.05 & 0.03 \\
\hline Subjective well-being & -0.15 & -1.12 & 0.81 & 0.05 & 0.93 & 0.10 & 1.75 & 0.03 \\
\hline
\end{tabular}


PGSs, but they were not significant ( $p \geq 0.278)$. The PGSs explained from 2 to $6 \%$ of the SOC variation.

Finally, we conducted the genome-wide scan of SOC on 1257 twin individuals. This analysis revealed four genomewide significant associations, all nearby on chromosome 2, for the following SNPs (rs74920024, rs72893369, rs 148317278 , rs72893377) with $p$ values from $2.034 \mathrm{e}-09$ to $3.300 \mathrm{e}-09$. The rs 74920024 reference allele is $\mathrm{G}$, while the minor allele frequency of the A-allele is 0.02277 . The Q-Q plot is shown in Fig. 1a and the Manhattan plot in Fig. 1b. The locus zoom plot (Fig. 1c) shows the locally correlated SNPs, but after conditioning on the top SNP, no further genome-wide signals were seen. The closest gene is glycerol-3-phosphate dehydrogenase 2 (GPD2). The GWA catalog reports no associations for the top SNP (https://www. ebi.ac.uk/gwas/home), but there are hits close by (Fig. 1c).

\section{Discussion}

Our results based on a Finnish sample of twins demonstrated that genetic factors explained a moderate proportion of individual differences in SOC, mental health, self-esteem and personality. These estimates corresponded well with the previous studies. Generally similar heritability estimates of personality [40] as well as mental health indicators used in this study, such as alexithymia [41] and depressive symptoms [42], have been previously reported. The previously reported heritability of SOC in a larger cohort of twins from which this cohort was derived was also very similar [19]. These results thus support the robustness of the genetic architecture of these psychological traits in adulthood with moderate heritability.

We found that SOC was associated with mental health indicators, self-esteem and personality traits. SOC was negatively associated with depression, alexithymia, schizotypal personality and overall mental health problems and positively associated with self-esteem. Previous studies have reported positive associations of SOC with life satisfaction [8] and negative associations with depression and anxiety [7, 8]. Additionally, for personality, the results were similar to those of previous studies. A recent meta-analysis of 24 independent samples (19,960 participants) using the NEO FFI personality scale found that, in accordance with our study, neuroticism was associated with weaker and extraversion, agreeableness and conscientiousness with stronger SOC.
A

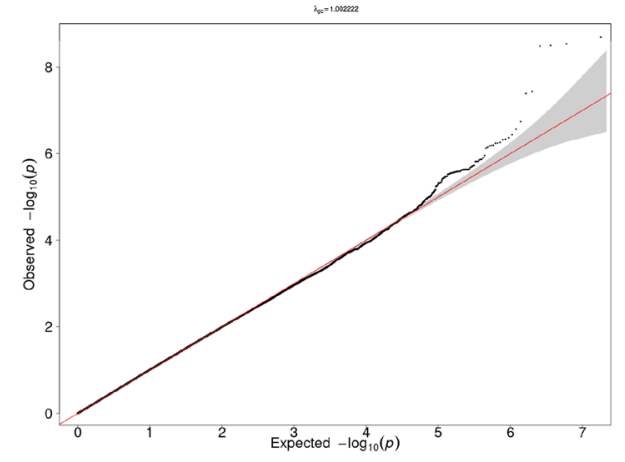

B

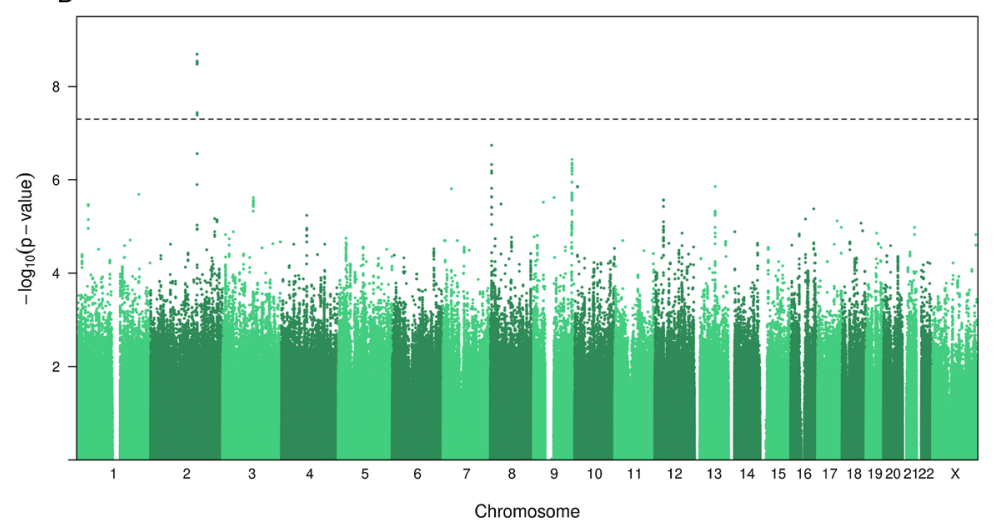

$$
\text { SOC_chr2 }
$$

C

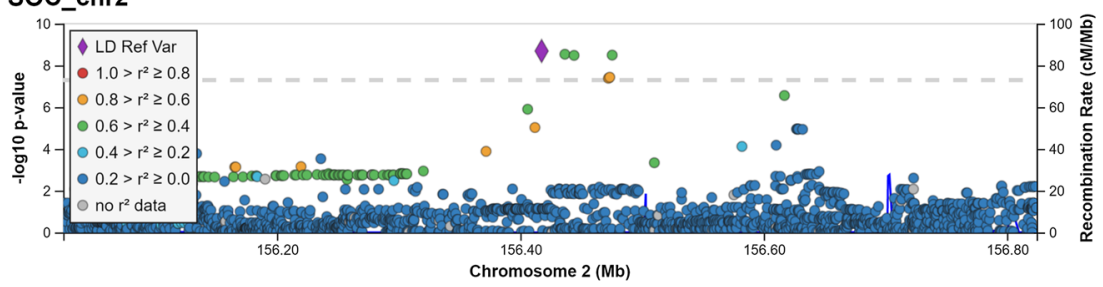

Hits in GWAS Catalog

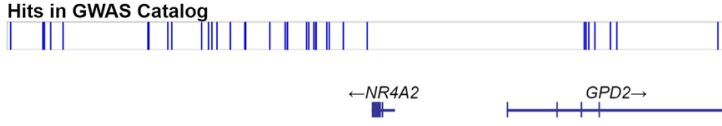

Fig. 1 The genome-wide scan of sense of coherence. a Quantilequantile plot of all $p$ values from variants passing quality control with a $45^{\circ}$ reference line plotted. A genomic inflation factor of $\lambda=1.00$ shows no inflation in $p$ values caused by population stratification or sample relatedness. b Manhattan plot of $p$ values from variants passing quality control. Horizontal line indicates genome-wide significant threshold for $p$ values (5e-08). c Regional plot of chromosome 2 results showing linkage disequilibrium between lead-SNP (rs74920024) and other variants within the zoomed region. The plot was generated with LocusZoom (https://my.locuszoom.org/) 
This meta-analysis also reported that openness was associated with stronger SOC, a finding we could not replicate; however, this correlation was substantially lower than correlations with other personality traits [9].

Our most novel results concerned the role of genetic factors behind the associations of SOC with the other psychological traits. Our results based on the genetic twin design revealed that even when both genetic and unique environmental factors contributed to these associations, the genetic correlations were generally stronger than unique environmental correlations. There was substantial variation in the size of genetic correlations, but they showed that from 30 to $60 \%$ of the genetic variation of most mental health indicators, self-esteem and personality traits was shared with SOC. On the other hand, this also shows that since the proportions are much less than $100 \%$, there are also genetic factors affecting these traits independently. The role of genetic factors was confirmed by the molecular genetic results showing that the PGSs of mental health indicators were correlated with SOC. The variation of SOC explained by the PGSs was $6 \%$ or less. However, this much lower share of genetic variation than found when using genetic twin modeling is expected since the PGSs do not yet cover the whole genome level variation and subsequently explain only a fraction of the genetic variation of mental health indictors estimated by genetic twin modeling, a phenomenon known as missing heritability [43]. For example, the heritability of neuroticism based on the PGS (called as SNP heritability) is only 0.11 (http://www.nealelab.is/uk-biobank), while the heritability estimates of neuroticism were 0.43 in men and 0.58 in women in our data estimated using the genetic twin modeling. Considering the difference between SNP heritability and heritability estimated based on genetic twin modeling, our results on shared genetic variation based on PGSs and genetic twin modeling are consistent. Since these two methods to estimate genetic variance are based on totally different theoretical assumptions, together they provide strong evidence for shared genetic effects between SOC and a range of mental health and personality traits.

This evidence of overlap in genetic liability to individual variation in SOC with psychological well-being, dimensions of mental health and personality creates opportunities for further research. The search for endophenotypes that mediate effects of dispositional genes on observed phenotypes of psychopathology [44] can be extended to endophenotypic pathways for risk-relevant behavioral precursors as illustrated by a study suggesting a novel metabolic biomarker, ketone body 3-hydroxybutyrate, of aggression [45]. Such research is responsive to the call to create a holistic framework for enhanced understanding of mental health traits, proposed by the National Institute of Mental Health's Research Domain Criteria (RDoC) Initiative [46]. Another part of the holistic framework creation goal of the RDoC initiative would be the elaboration of models of well-being [47]. Our results suggest the possibility of identifying biobehavioral processes that underlie broad dimensions of psychological health, ranging from processes creating high risk for psychiatric disorders to less severe, but more common behavioral and adjustment problems, to individual differences in resilience to stress, self-efficacy, and positive psychological health. Identifying possible endophenotypes underlying diverse mental health traits and effectively fostering dimensional research on the biobehavioral matrix of mental health and mental illness is a challenging new opportunity for multidisciplinary research.

Our GWA analysis, to our knowledge the first one conducted for SOC, yielded a significant association ( $p=2.034 \mathrm{e}-09$ for $\mathrm{rs} 74920024$ on chromosome 2 ), with three other significant associations, though just one independent signal. While our study is a priori underpowered with a sample size of 1257 , the use of a continuous variable and the more powerful TOPMed reference panel may have partly compensated for this problem. The association locus is near but not in the closest gene, GPD2. The gene encodes a protein that localizes to the inner mitochondrial membrane and is responsible for the conversion of glycerol-3-phosphate to dihydroxyacetone phosphate. Interestingly, it has been associated in several large GWA analyses with education [48] and cognitive ability [49] in addition to myopia [50], a condition known to be aggravated by reading. However, because of the small sample size for the GWA, our results should be considered as only preliminary and require replications in other datasets.

There can be different mechanisms explaining the genetic correlations found. First, they can reflect the influence of genes on different traits (called pleiotropy). Second, they can be because of causal associations between the traits. For example, if strong SOC is causally associated with better self-esteem, it can create a genetic correlation when genetic polymorphisms increasing SOC also explain better mental health. However, the fact that genetic correlations were systematically higher than the trait correlations does not support this explanation. Third, the genetic correlations can indicate the same neurobiological background behind these indicators. We found this explanation most plausible, and it is consistent with the theory that SOC largely captures the same dimension of mental health as other known indicators of mental health [51].

Since the prevalence of internalized mental health disorders is higher in women than in men [52], we stratified all analyses by sex to study whether sex differences may also exist in the associations between SOC and other psychological traits. We found that, except alexithymia, men had better mental health and also higher self-esteem than women. Additionally, SOC was stronger in men than in women, as also found in previous studies $[53,54]$. Consistent with previous studies [55], men had higher levels of extraversion and 
women higher levels of neuroticism, openness and agreeableness. Considering these mean differences, it is interesting that the associations of SOC with mental health, self-esteem and personality traits were very similar in men and women, and we did not find any systematic sex differences in the genetic correlations either. When using PGSs, some sex differences were found, but they were not statistically significant and, therefore, can also be because of sample error. Thus, based on this study, the genetic background of SOC and other psychological traits seems to be broadly similar in men and women.

Our study has both strengths and weaknesses. Our main strengths were that we had information on SOC and several mental health, well-being and personality indicators measured with well-validated questionnaires in genetically informative data. We were able to study the common genetic background of SOC, mental health, self-esteem and personality using two different approaches-the classical twin design utilizing information on the similarity of MZ and DZ twins and the molecular genetic design utilizing measured genetic polymorphisms-each making different theoretical assumptions. The robustness of these results provides more convincing evidence on the role of genes behind these associations. A limitation is that we needed to rely on selfreported data, without information on clinically validated psychiatric diseases or ratings of personality factors by others. Thus, a response bias may have strengthened trait correlations, but they are not likely to explain our main results (i.e., the genetic correlations between the traits). Finally, it is clear that our data are underpowered for GWA; thus, these results need to be considered as preliminary, needing replications in other datasets.

In conclusion, we found that SOC shares a substantial proportion of genetic variation with mental health indicators, self-esteem and personality factors. This indicates that the correlations between these traits reflect in part a common neurobiological background rather than the influence of external stressors. Our results may suggest that mental health forms a continuum from mental health disorders of various severity to the positive end of mental health and well-being. Further research is needed to find neurobiological mechanisms behind the whole variation of mental health. Our results also suggest that mental health treatment may benefit a larger share of the population than only those with diagnosed mental health disorders.

Supplementary Information The online version contains supplementary material available at https://doi.org/10.1007/s00127-021-02098-6.

Author contributions KS conducted the analyses and drafted the manuscript. SMV and SS helped to draft the sections related to the theory of sense of coherence. TP created the polygenic risk scores and conducted the genome-wide-association analyses of sense of coherence. JK and RJR collected the data. EV, SMV, TP RJR, SS and JK contributed to the interpretation of data and critically revised the manuscript. All authors approve the version to be published and agree to be accountable for all aspects of the work.

Funding Open access funding provided by University of Helsinki including Helsinki University Central Hospital. Data collections and genotyping has been supported by National Institute of Alcohol Abuse and Alcoholism (Grants AA-12502, AA-00145, and AA-09203 to RJR and AA15416 and K02AA018755 to Danielle M Dick) and the Academy of Finland (grants 100499, 205585, 118555, 141054, 264146, 308248 and 312073 to JK).

\section{Declarations}

Conflict of interest On behalf of all authors, the corresponding author states that there are no conflicts of interest.

Ethics approval The ethics committee of the Department of Public Health of the University of Helsinki (Helsinki, Finland), the ethics committee of the Helsinki University Central Hospital District (Helsinki, Finland), and the Institutional Review Board of Indiana University (Bloomington, Indiana, USA) approved the FinnTwin12 study protocol.

Consent to participate All participants and their parents/legal guardians gave informed written consent to participate in the study.

Consent for publication All authors accept the publication of this article.

Availability of data and materials Because of the consent given by study participants and the high degree of identifiability, data cannot be made publicly available. Data are available through the Institute for Molecular Medicine Finland (FIMM) Data Access Committee (DAC) for authorized researchers who have IRB/ethics approval and an institutionally approved study plan. For more details, please contact the FIMM DAC (fimm-dac@ helsinki.fi).

Code availability All computer codes are available from the corresponding author.

Open Access This article is licensed under a Creative Commons Attribution 4.0 International License, which permits use, sharing, adaptation, distribution and reproduction in any medium or format, as long as you give appropriate credit to the original author(s) and the source, provide a link to the Creative Commons licence, and indicate if changes were made. The images or other third party material in this article are included in the article's Creative Commons licence, unless indicated otherwise in a credit line to the material. If material is not included in the article's Creative Commons licence and your intended use is not permitted by statutory regulation or exceeds the permitted use, you will need to obtain permission directly from the copyright holder. To view a copy of this licence, visit http://creativecommons.org/licenses/by/4.0/.

\section{References}

1. Ryan RM, Deci EL (2001) On happiness and human potentials: a review of research on hedonic and eudaimonic well-being. Annu Rev Psychol 52:141-166

2. Antonovsky A (1987) Unraveling the Mystery of Health. JosseyBass, How People Manage Stress and Stay Well. San Francisco 
3. Eriksson M, Lindström B (2006) Antonovsky's sense of coherence scale and the relation with health: a systematic review. J Epidemiol Community Health 60:376-381

4. Eriksson M, Lindström B (2007) Antonovsky's sense of coherence scale and its relation with quality of life: a systematic review. J Epidemiol Community Health 61:938-944

5. Eriksson M, Lindström B (2005) Validity of Antonovsky's sense of coherence scale: a systematic review. J Epidemiol Community Health 59:460-466

6. Antonovsky A (1993) The structure and properties of the sense of coherence scale. Soc Sci Med 36:725-733

7. Konttinen H, Haukkala A, Uutela A (2008) Comparing sense of coherence, depressive symptoms and anxiety, and their relationships with health in a population-based study. Soc Sci Med 66:2401-2412

8. Pallant JF, Lae L (2002) Sense of coherence, well-being, coping and personality factors: further evaluation of the sense of sense of coherence scale. Personality Individ Differ 33:39-48

9. Barańczuk U (2021) The five factor model of personality and sense of coherence: a meta-analysis. J Health Psychol 26:12-25

10. Volanen SM, Suominen S, Lahelma E, Koskenvuo M, Silventoinen K (2007) Negative life events and stability of sense of coherence: a five-year follow-up study of Finnish women and men. Scand J Psychol 48:433-441

11. Masanotti GM, Paolucci S, Abbafati E, Serratore C, Caricato M (2020) Sense of coherence in nurses: a systematic review. Int J Environ Res Public Health 13:E1861

12. Brainstorm Consortium (2018) Analysis of shared heritability in common disorders of the brain. Science 360(6395):eaap8757

13. Cross-Disorder Group of the Psychiatric Genomics Consortium (2019) Genomic relationships, novel loci, and pleiotropic mechanisms across eight psychiatric disorders. Cell 179:1469-1482

14. Okbay A, Baselmans BM, de Neve JE, Turley P, Nivard MG, Fontana MA et al (2016) Genetic variants associated with subjective well-being, depressive symptoms, and neuroticism identified through genome-wide analyses. Nat Genet 1:624-633

15. Baselmans BML, Jansen R, Ip HF, van Dongen J, Abdellaoui A, van de Weijer MP et al (2019) Multivariate genome-wide analyses of the well-being spectrum. Nat Genet 51:445-451

16. Kaprio J, Pulkkinen L, Rose RJ (2002) Genetic and environmental factors in health-related behaviors: studies on Finnish twins and twin families. Twin Res 5:366-371

17. Rose RJ, Salvatore JE, Aaltonen S, Barr PB, Bogl LH, Byers HA et al (2019) FinnTwin 12 cohort: an updated review. Twin Res Hum Genet 22:302-311

18. Jelenkovic A, Ortega-Alonso A, Rose RJ, Kaprio J, Rebato E, Silventoinen K (2011) Genetic and environmental influences on growth from late childhood to adulthood: a longitudinal study of two Finnish twin cohorts. Am J Hum Biol 23:764-773

19. Silventoinen K, Volanen SM, Vuoksimaa E, Rose RJ, Suominen S, Kaprio J (2014) A supportive family environment in childhood enhances the level and heritability of sense of coherence in early adulthood. Soc Psychiatry Psychiatr Epidemiol 49:1951-1960

20. Ranjit A, Korhonen T, Buchwald J, Heikkilä K, Tuulio-Henriksson A, Rose RJ et al (2019) Testing the reciprocal association between smoking and depressive symptoms from adolescence to adulthood: a longitudinal twin study. Drug Alcohol Depend 200:64-70

21. Bagby RM, Parker JDA, Taylor GJ (1994) The twenty-item Toronto Alexithymia Scale-I. Item selection and cross-validation of the factor structure. J Psychosom Res 38:23-32

22. Raine A, Benishay D (1995) The SPQ-B: a brief screening instrument for schizotypal personality disorder. J Personal Disord 9:346-355
23. Penninkilampi-Kerola V, Miettunen J, Ebeling H (2006) A comparative assessment of the factor structures and psychometric properties of the GHQ-12 and the GHQ-20 based on data from a Finnish population-based sample. Scand J Psychol 47:431-440

24. Rosenberg M (1965) Society and the Adolescent Self-image. Princeton University Press, Princeton

25. Raevuori A, Dick DM, Keski-Rahkonen A, Pulkkinen L, Rose RJ, Rissanen A et al (2007) Genetic and environmental factors affecting self-esteem from age 14 to 17: a longitudinal study of Finnish twins. Psychol Med 37:1625-1633

26. McCrae RR, Costa PTJ (2003) Personality in Adulthood: A Five-Factor Theory Perspective, 2nd edn. Guilford Press, New York

27. Pulver A, Allik J, Pulkkinen L, Hämäläinen M (1995) The Big Five Personality Inventory in two non-Indo-European languages. Eur J Pers 9:109-124

28. Posthuma D, Beem AL, de Geus EJ, van Baal GC, von Hjelmborg JB, Iachine I et al (2003) Theory and practice in quantitative genetics. Twin Res 6:361-376

29. Kujala UM, Palviainen T, Pesonen P, Waller K, Sillanpää E, Niemelä M et al (2020) Polygenic risk scores and physical activity. Med Sci Sports Exerc 52:1518-1524

30. Vilhjálmsson BJ, Yang J, Finucane HK, Gusev A, Lindström S, Ripke $S$ et al (2015) Modeling linkage disequilibrium increases accuracy of polygenic risk scores. Am J Hum Genet 97:576-592

31. Borodulin K, Vartiainen E, Peltonen M, Jousilahti P, Juolevi A, Laatikainen $\mathrm{T}$ et al (2015) Forty-year trends in cardiovascular risk factors in Finland. Eur J Public Health 25:539-546

32. Wray NR, Ripke S, Mattheisen M, Trzaskowski M, Byrne EM, Abdellaoui A et al (2018) Genome-wide association analyses identify 44 risk variants and refine the genetic architecture of major depression. Nat Genet 50:668-681

33. Schizophrenia Working Group of the Psychiatric Genomics Consortium (2014) Biological insights from 108 schizophreniaassociated genetic loci. Nature 511:421-427

34. Karlsson Linnér R, Biroli P, Kong E, Meddens SFW, Wedow R, Fontana MA et al (2019) Genome-wide association analyses of risk tolerance and risky behaviors in over 1 million individuals identify hundreds of loci and shared genetic influences. Nat Genet $51: 245-257$

35. Taliun D, Harris DN, Kessler MD, Carlson J, Szpiech ZA, Torres $\mathrm{R}$ et al (2021) Sequencing of 53,831 diverse genomes from the NHLBI TOPMed Program. Nature 590:290-299

36. Fuchsberger C, Abecasis GR, Hinds DA (2014) Minimac2: faster genotype imputation. Bioinformatics 31:782-784

37. Das S, Forer L, Schönherr S, Sidore C, Locke AE, Kwong A et al (2016) Next-generation genotype imputation service and methods. Nat Genet 48:1284-1287

38. Neale MC, Hunter MD, Pritikin JN, Zahery M, Brick TR, Kirkpatrick RM et al (2016) OpenMx 2.0: Extended structural equation and statistical modeling. Psychometrika 81:535-549

39. Williams R (2000) A note on robust variance estimation for cluster-correlated data. Biometrics 56:645-646

40. Vukasovic T, Bratko D (2015) Heritability of personality: a metaanalysis of behavior genetic studies. Psychol Bull 141:769-785

41. Picardi A, Fagnani C, Gigantesco A, Toccaceli V, Lega I, Stazi MA (2011) Genetic influences on alexithymia and their relationship with depressive symptoms. J Psychosom Res 71:256-263

42. Agrawal A, Jacobson KC, Gardner CO, Prescott CA, Kendler KS (2004) A population based twin study of sex differences in depressive symptoms. Twin Res 7:176-181

43. Young AI (2019) Solving the missing heritability problem. PLoS Genet 15:e1008222

44. Gottesman II, Gould TD (2003) The endophenotype concept in psychiatry: etymology and strategic intentions. Am J Psychiatry 160:636-645 
45. Whipp AM, Vuoksimaa E, Korhonen T, Pool R, Ligthart RSL, Hagenbeek FA et al (2021) Ketone body 3-hydroxybutyrate as a biomarker of aggression. Sci Rep 11:5813

46. Carcone D, Ruocco AC (2017) Six years of research on the National Institute of Mental Health's Research Domain Criteria (RDoC) Initiative: a systematic review. Front Cell Neurosci. https://doi.org/10.3389/fncel.2017.00046

47. Ryff CD, Keyes CLM (1995) The structure of psychological wellbeing revisited. J Pers Soc Psychol 69:719-727

48. Kichaev G, Bhatia G, Loh PR, Gazal S, Burch K, Freund MK et al (2019) Leveraging polygenic functional enrichment to improve GWAS Power. Am J Hum Genet 104:65-75

49. Lam M, Trampush JW, Yu J, Knowles E, Davies G, Liewald DC et al (2017) Large-scale cognitive GWAS meta-analysis reveals tissue-specific neural expression and potential nootropic drug targets. Cell Rep 21:2597-2613

50. Hysi PG, Choquet H, Khawaja AP, Wojciechowski R, Tedja MS, Yin J et al (2020) Meta-analysis of 542,934 subjects of European ancestry identifies new genes and mechanisms predisposing to refractive error and myopia. Nat Genet 52:401-407

51. Geyer S (1997) Some conceptual consideration on the sense of coherence. Soc Sci Med 44:1771-1779
52. Seedat S, Scott KM, Angermeyer MC, Berglund P, Bromet EJ, Brugha TS et al (2009) Cross-national associations between gender and mental disorders in the World Health Organization World Mental Health Surveys. Arch Gen Psychiatry 66:785-795

53. Anson O, Paran E, Neumann L, Chernichovsky D (1993) Gender differences in health perceptions and their predictors. Soc Sci Med 36:419-427

54. Larsson G, Kallenberg K (1996) Sense of coherence, socio-economic conditions and health. Eur J Public Health 6:175-180

55. Costa PT Jr, Terracciano A, McCrae RR (2001) Gender differences in personality traits across cultures: robust and surprising findings. J Pers Soc Psychol 81:322-331 